\title{
ПРИМЕНЕНИЕ РАЗЛИЧНЫХ ИНДЕКСОВ ИНСУЛИНОРЕЗИСТЕНТНОСТИ У ПАЦИЕНТОВ С МЕТАБОЛИЧЕСКИМ СИНДРОМОМ ПРИ АССОЦИАЦИИ С ПОЛИМОРФИЗМОМ ГЕНА PPAR-GAMMA RS1801282
}

\author{
Еременко Т.В., Ворохобина Н.В., Матезиус И.Ю., Мациевский Н.А., Поташев Т.А. \\ ФГБОУ ВО «СЗГМУ им. И. И. Мечникова» Минздрава России, Санкт-Петербург
}

\begin{abstract}
ЦЕЛЬ: установить взаимосвязь показателей инсулинорезистентности, рассчитанной различными формулами с компонентами метаболического синдрома у пациентов при ассоциации с полиморфизмом гена PPAR-gamma rs1801282
\end{abstract}

МАТЕРИАЛЫ И МЕТОДЫ: в исследование были включены 145 пациентов с впервые выявленным метаболическим синдромом. Исследуемую группу составили 87 (60,0\%) мужчин и 58 (40,0\%) женщин, в возрасте 52 [49; 57] года. Индекс массы тела составил 34 [32;36] кг/м². Пациентам была определена гликемия натощак, инсулинемия натощак, показатели липидного спектра крови, рассчитаны индексы инсулинорезистентности. Для определения индексов инсулинорезистентности использовались следующие формулы: HOMA-IR, QUICKI, McAuley, Raynaud, IGR, ISIb Belfiore.

Всем пациентам проводили генотипирование полиморфизма гена PPAR-gamma rs1801282 стандартным методом - амплификация фрагментов ДНК методом полимеразно-цепной реакции. На основании результатов генотипирования пациенты были разделены на 2 группы наблюдения с учетом наличия полиморфизма.

PЕзУЛЬтАТЫ: у 35 (24,1\%) участников определено 3 критерия метаболического синдрома, у 46 (31,7\%) - 4, у 64 (44,1\%) - 5. Все обследованные пациенты характеризовались высоким уровнем инсулинорезистентности согласно рассчитанным индексам, так, HOMA-IR составил 7,66 [5,77; 9,83], QUICKI - 0,29 [0,28; 0,30], McAuley 4,31 [3,92; 4,60], Raynaud 1,43 [1,25; 1,67], IGR 4,37 [3,21; 5,32]; ISIb $58,05[45,21 ; 77,04]$; Belfiore 0,51 [0,42; 0,62]. В группе пациентов с установленным полиморфизмом rs1801282 обнаружены более высокие показатели индексов инсулинорезистентности QUICKI, Raynaud, ISIb, Belfiore. Индекс McAuley не показал различий при сравнении популяций пациентов с полиморфизмом rs1801282 и без нее. Проведен корреляционный анализ рассчитанных индексов с основными параметрами течения метаболического синдрома, отличными от параметров гликемии и инсулинемии, на основании которых указанные индексы рассчитываются. Не выявлено значимой корреляции ( $r>0,3$, p <0,05) индексов с индексом массы тела, окружностью талии, уровнями общего холестерина и липопротеидов низкой плотности. Обнаружена корреляция индексов HOMA-IR, QUICKI, McAuley, ISIb c ypoвнем триглицеридов ( $r$ составил -0,33, 0,33, -0,60, -0,33, 0,33 соответственно). Значимая корреляция интегративного показателя суммы критериев метаболического синдрома у пациента обнаружена только с индексом McAuley (r=-0,31). Проведенное исследование указывает на сопоставимое значение у ряда индексов инсулинорезистентности (HOMA-IR, QUICKI, Raynaud, ISIb, Belfiore) для пациентов с метаболическим синдромом.

ВЫВОДЫ: результаты настоящего исследования свидетельствуют о целесообразности использования в популяции пациентов с метаболическим синдромом для изучения инсулинорезистентности индексов HOMA-IR, QUICKI, McAuley и ISIb.

Получены данные о том, что индекс McAuley имеет наилучшую корреляцию с интегративным показателем суммы критериев МС у пациентов. 